\title{
Searching for merger debris in the Galactic halo: chemodynamical evidence based on local blue HB stars
}

\author{
M. Altmann ${ }^{1}$, M. Catelan ${ }^{2}$, and M. Zoccali ${ }^{2}$ \\ ${ }^{1}$ Departamento de Astronomía, Universidad de Chile, Casilla 36-D, Correo Central, Santiago, Chile \\ e-mail: martin@das.uchile.cl \\ 2 Pontificia Universidad Católica de Chile, Departamento de Astronomía y Astrofísica, Av. Vicuña Mackenna 4860, \\ 782-0436 Macul, Santiago, Chile \\ e-mail: [mcatelan;mzoccali]@astro.puc.cl
}

Received 1 February 2005 / Accepted 26 June 2005

\begin{abstract}
We report on the discovery of a group of local A-type blue horizontal-branch (HBA) stars moving in a prograde, comet-like orbit with very similar kinematics and abundances. This serendipitously discovered group contains 5 or 6 local HBA stars venturing very close to the Galactic centre; their $[\mathrm{Fe} / \mathrm{H}]$ is around -1.7 , and they seem to present minimum scatter in at least $\mathrm{Mg}, \mathrm{Si}, \mathrm{Ti}, \mathrm{Fe}, \mathrm{Al}$, and $\mathrm{Cr}$ abundances. This "Cometary Orbit Group" (COG) was found while we were testing a new method to detect the debris associated with the merger of smaller, specific protogalactic entities into our galaxy. The method is primarily intended to identify field HBA stars with similar kinematics and detailed, multi-species abundance patterns as seen among members of a surviving remnant (e.g., $\omega$ Centauri). Quite possibly, the COG is the remnant, on a highly decayed orbit, of a merging event that took place in the relatively remote past (i.e., at least one revolution ago).
\end{abstract}

Key words. astrometry - stars: kinematics - stars: horizontal branch - Galaxy: halo - Galaxy: structure

\section{Introduction}

Over the past decade evidence has accumulated that the Galactic halo was at least partially built up by smaller entities that merged with the main body of the Galaxy. Particularly compelling evidence in this direction has been provided by the discovery of the Sagittarius dwarf spheroidal (dSph) galaxy (Ibata et al. 1994). More recently, another object penetrating our galaxy has been found, namely the Canis Major dwarf galaxy (Martin et al. 2004). One object which has been repeatedly mentioned as the possible remnant of a merger process is $\omega$ Centauri (NGC 5139), the most massive Galactic globular cluster (GC). Its unique properties among GCs, including a large spread in metalicities (Norris \& Bessel 1975) and ages (Hilker et al. 2004 and references therein), have raised suspicions that $\omega$ Cen may in fact be the nucleus of a former dwarf galaxy which was accreted into the main body of the Milky Way several Gyr ago. This hypothesis is strengthened by the fact that the second most massive Galactic GC, M 54 (NGC 6715), is in fact associated with the Sagittarius dSph, and may even constitute its nucleus (e.g., Layden $\&$ Sarajedini 2000, and references therein).

In the present Letter, we report on the discovery of a group of stars that seems likely to be associated with a merger event. This group, which we will refer to as the Cometary Orbit Group (COG), was found while we were testing a new method, involving detailed orbital information and multi-species element abundances for A-type blue horizontal branch (HBA) stars, devised to identify tidal debris related to a specific surviving protogalactic fragment, such as $\omega$ Cen itself.

\section{The method}

Several studies have attempted to identify the debris of an $\omega$ Cen-related merger event among field stars (Dinescu 2002; Bekki \& Freeman 2003; Mizutani et al. 2003). All of these predict the remnant moving in a retrograde manner (as $\omega$ Cen itself). Our primary goal, with the proposed method, is to identify local HBA stars moving in a retrograde orbit $(\Theta<$ $0 \mathrm{~km} \mathrm{~s}^{-1}$ ) with abundance patterns that are similar to those seen among evolved red giant branch (RGB) stars in $\omega$ Cen, thus providing a chemodynamical signature of their prior membership to this cluster.

Why focus on HBA stars instead of, say, red HB/red clump stars, RR Lyrae stars, or RGB stars? First of all, HBA stars are almost exclusively associated with the Galactic halo, unlike red $\mathrm{HB} /$ red clump and RGB stars, which comprise a much more heterogeneous mix and whose properties are much more difficult to disentangle from one another. Also, while $\omega$ Cen possesses a vast population of HBA stars, it is almost devoid of red HB/red clump stars (see, e.g., Fig. 1 in Ferraro et al. 2004). Moreover, HBA stars being non-variable, their spectra are much easier to interpret than in the case of RR Lyrae 
variables. Last but not least, B-type blue HB (BHB) stars show strong abundance anomalies which do not reflect the abundances they had by the time they reached the RGB tip, being due instead to gravitational diffusion and radiative levitation (Moehler 2004, and references therein).

HB stars are the immediate progeny of RGB tip stars. As such, one expects to find a close similarity between photospheric abundances for RGB tip stars and HBA stars belonging to the same population. This implies that field HBA stars which have once belonged to $\omega$ Cen should have unique signatures that would clearly set them apart from other HBA stars, due to the fact that RGB stars in $\omega$ Cen are well known to have peculiar abundance patterns in $\mathrm{O}, \mathrm{Na}, \mathrm{Al}, \mathrm{Mg}, \mathrm{Cu}, \mathrm{Eu}$, and the s-process elements, including extreme over- and underabundances compared to field stars with similar $[\mathrm{Fe} / \mathrm{H}]$ (some of the differences being themselves a function of $[\mathrm{Fe} / \mathrm{H}]$; e.g., Norris \& Da Costa 1995; Smith et al. 2000; Pancino et al. 2002). Such abundance patterns are not only unparallelled in other GCs; they have actually never been found among field halo stars (e.g., Gratton et al. 2000). In this sense, it would be extremely unlikely for a field star moving in a retrograde orbit with abundances $[\mathrm{O} / \mathrm{Fe}] \leq-0.4,[\mathrm{Al} / \mathrm{Fe}] \geq+0.75,[\mathrm{Mg} / \mathrm{Fe}] \geq+0.6$, $[\mathrm{Cu} / \mathrm{Fe}] \leq-0.5,[\mathrm{Eu} / \mathrm{Fe}] \leq+0.1,[\mathrm{Ba} / \mathrm{Fe}] \geq+0.75,[\mathrm{La} / \mathrm{Fe}] \geq$ +0.75 not to have once been associated with $\omega$ Cen. Therefore, identifying HBA stars in the halo field with kinematics and detailed abundance signatures which are both consistent with former membership in the cluster would provide compelling evidence of the presence of $\omega$ Cen debris in the field.

\section{Sample composition and data}

Our sample consists of all known and unambiguously classified local ( $d \leq 1 \mathrm{kpc}$ ) HBA stars for which the required data, such as radial velocities and proper motions, are available. We restricted ourselves to HBA stars, i.e. blue HB stars with a temperature of less than $10500 \mathrm{~K}$, since hotter HB stars show strong peculiarities in their abundances due to radiative levitation and diffusion processes in their atmospheres (Moehler 2004, and references therein), thus not allowing access to the envelope abundances of their immediate progenitors (RGB tip stars). We took all HBA stars from Altmann \& de Boer (2000, hereafter AdB00), and added the bona-fide HBA stars from Kinman et al. (2000, hereafter K00) and Behr (2003, hereafter B03). A total of 30 stars made it into our final sample, more than twice the number in AdB00.

All the relevant data were taken from the literature. Most of the stars have Hipparcos (ESA 1997) parallaxes and proper motions, except in a few cases, where we had to rely on the slightly less accurate Tycho2 (Høg et al. 2000) proper motions. Since the parallaxes are mostly too inaccurate to directly derive the distances, we chose the approach used in AdB00, to which we refer the interested reader for further details.

The radial velocities were also taken from AdB00, K00 and B03. Comparison among the different sources shows a generally good agreement, with the exception of HD 117880 where the value used in AdB00 (taken from Evans 1967) differs by almost $200 \mathrm{~km} \mathrm{~s}^{-1}$ from the other values. Since our own lowresolution spectra show radial velocities rather similar to K00 and B03, we conclude that the velocity from Evans must be incorrect. For the errors in the kinematics we quote the values given in AdB00, of about $10 \mathrm{~km} \mathrm{~s}^{-1}$ on average, with some dependence on distance.

Our primary source for abundances, $T_{\text {eff }}$, and $\log g$ is $\mathrm{K} 00$. Not only is this study more complete than B03, it also has more element species than just $\mathrm{Mg}$ and $\mathrm{Fe}$. While for those stars which are included in both studies the general agreement for $[\mathrm{Fe} / \mathrm{H}], T_{\text {eff }}, \log g$ is quite good, there is the exception of HD 117880, for which the results of B03 differ significantly from those of $\mathrm{K} 00$ and other sources in the literature. K00 have $[\mathrm{Mg} / \mathrm{H}]$ values for all of their programme stars, $[\mathrm{Fe} / \mathrm{H}]$ and $[\mathrm{Ti} / \mathrm{H}]$ for $\operatorname{most}^{1}$ and $[\mathrm{Ba} / \mathrm{H}]$ for a few. For some objects, we were able to include abundances of some additional elements from Adelman \& Philip (1996) (and their earlier work).

The proper motions, radial velocities, distances, etc. were converted to the $X Y Z U V W \Theta \Phi$ coordinate system and orbits calculated using the potential of Allen \& Santillan (1991), as described in more detail in AdB00 and Altmann et al. (2004). One of the quantities derived is $I_{z}$, the angular momentum in the $z$-direction ${ }^{2}$, representing the orbital motion in the Galactic plane. In contrast to the related orbital velocity $\Theta$, this is a conserved quantity - and plays a crucial role in the following analysis. The velocity errors translate into an error of $80 \mathrm{kpc} \mathrm{km} \mathrm{s}^{-1}$ for $I_{z}$ at the position of the Sun.

\section{Results}

The new, enlarged sample confirms AdB00's basic results - all HBA stars belonging to the halo. This can clearly be seen in the Toomre diagram shown in Fig. 1, especially in comparison with similar diagrams in Altmann et al. (2004) and Kaempf et al. (2005). One or two stars could in principle belong to the lowvelocity end of the thick disk, but since thick disk stars with more "normal" orbital velocities are absent and given the fact that there are stars with rather similar but retrograde velocities, we conclude that we are still dealing with a pure halo sample, even more so since none of the potential thick disk candidates is particularly metal-rich.

Two stars have orbital velocities significantly larger than $\Theta_{\mathrm{LSR}}$ (the disk velocity), one of which (HD 213468) with an extreme $\Theta=470 \mathrm{~km} \mathrm{~s}^{-1}$ and an apogalactic distance of over $200 \mathrm{kpc}$ ! We have thus found the first kinematic evidence of HBA stars belonging to the "high velocity halo," similar to the sdB stars found in Altmann et al. (2004) and objects like Barnard's star. For this reason, the average orbital velocity is $58 \mathrm{~km} \mathrm{~s}^{-1}$, compared to the $17 \mathrm{~km} \mathrm{~s}^{-1}$ found in AdB00. Removing these two stars results in $\bar{\Theta} \approx 35 \mathrm{~km} \mathrm{~s}^{-1}$. However, the dispersion $\sigma_{\Theta}$ is much higher in this new sample than the $55 \mathrm{~km} \mathrm{~s}^{-1}$ of AdB00, namely $80-120 \mathrm{~km} \mathrm{~s}^{-1}$, depending on whether the two "fast" stars are included or not. Note that the distribution of orbital velocities shows signs of bimodality, with the prograde sample seemingly separated from the retrograde

\footnotetext{
In a few cases, $[\mathrm{Fe} / \mathrm{H}]$ had to be derived from $[\mathrm{Mg} / \mathrm{H}]$. However, comparison between the $\mathrm{K} 00[\mathrm{Fe} / \mathrm{H}]$ values derived directly or via $[\mathrm{Mg} / \mathrm{H}]$ does not reveal noticeable systematic discrepancies.

2 Also denoted $J_{z}$ or $L_{z}$ in many publications.
} 


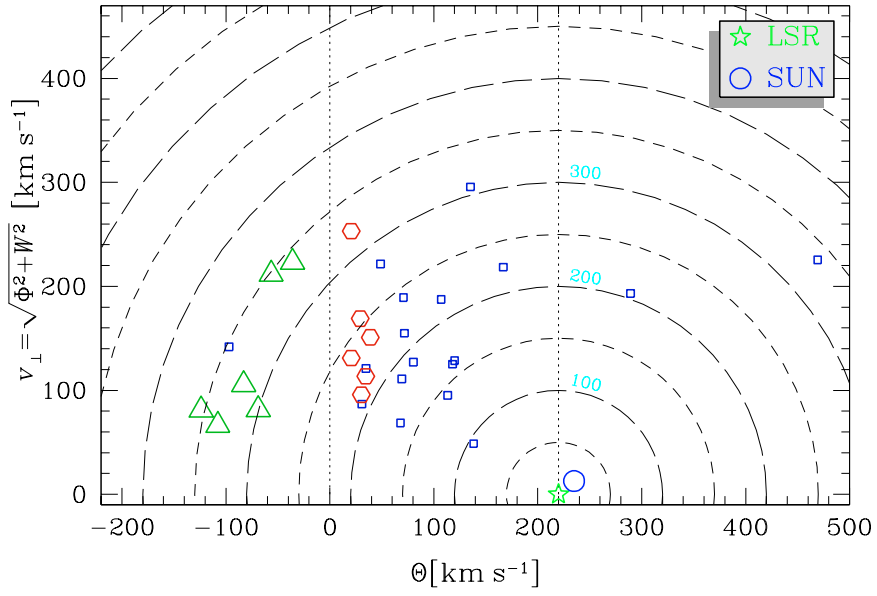

Fig. 1. Toomre diagram for the HBA stars in our sample. The concentric circles show the absolute peculiar velocity $\left(v_{\text {pec }}\right)$, i.e. the total deviation from circular velocity (shown as an open star; the Sun's position in this diagram is shown as an open circle). The hexagons depict the stars belonging to the members of the Cometary Orbit Group (COG) discussed in this paper, the triangles denote putative $\omega$ Cen debris candidates (identified as retrograde moving stars broadly conforming to the abundance range of $\omega$ Cen giants), the other HBAs are represented by squares. Note that the COG stars are relatively close together in this plane, their larger spread in $v_{\perp}$ most likely caused by their chaotic orbits. The most deviant point of this group is HD 86986. The rather large $\left(\geq 100 \mathrm{~km} \mathrm{~s}^{-1}\right) v_{\text {pec }}$ reveals that most probably all objects belong to the halo. Stars of the thin disk have a $v_{\text {pec }}$ of mostly less than $50 \mathrm{~km} \mathrm{~s}^{-1}$, while some thick disk stars may have a $v_{\text {pec }}$ of up to $150 \mathrm{~km} \mathrm{~s}^{-1}$.

group by a gap. Furthermore, we found evidence of substructure in the kinematics of our HBA stars, which may be related to the halo's merger history.

Seven of our stars $(23 \%)$ have retrograde orbits, similar to $\omega$ Cen itself $\left(\Theta=-69 \mathrm{~km} \mathrm{~s}^{-1}, I_{z}=-457 \mathrm{kpc} \mathrm{km} \mathrm{s}^{-1}\right)$. Bekki $\&$ Freeman (2003) and Tsuchiya et al. (2004) have calculated scenarios for the merger event forming the present-day $\omega$ Cen, predicting the kinematics of remnant stars in the solar vicinity. If these merger scenarios hold true, at least some of our retrograde stars could indeed represent debris from this merger event. However, as discussed in Sect. 2, a clear-cut signature of prior association can only be provided by a very detailed abundance analysis (see also Dinescu 2002).

\subsection{The Cometary Orbit moving group (COG)}

In the course of our search for $\omega$ Cen candidates, we serendipitously found a very striking group with common properties among our prograde stars. This group, consisting of four to six stars ${ }^{3}$, is located near $I_{z}=+230 \mathrm{kpc} \mathrm{km} \mathrm{s}^{-1}$ and $[\mathrm{Fe} / \mathrm{H}]=$ -1.7 , according to Fig. 2. In this figure, we plot the abundances of several different species as a function of $I_{z}$. The COG is very confined in $I_{z}\left(\sigma_{I_{z}}=55 \mathrm{kpc} \mathrm{km} \mathrm{s}{ }^{-1}\right)$ and in most element

${ }^{3}$ HD 106304 has abundances which are slightly discrepant with respect to the other COG stars and HD 86986 has somewhat different radial kinematics, see Fig. 1.
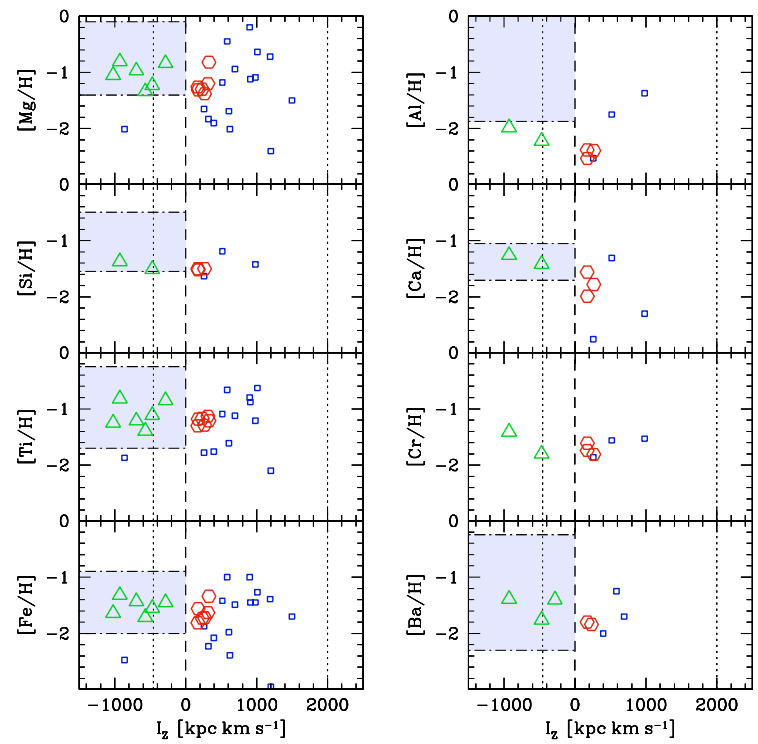

Fig. 2. $[\mathrm{X} / \mathrm{H}]$ vs. $I_{z}$ diagram for eight different elements. The vertical dashed line marks the boundary between pro- and retrograde rotation, while the dotted lines $\omega$ Cen's $I_{z}$ (left) and that of the Sun (right) to represent the $I_{z}$ of a typical disk star. The grey area between the horizontal lines in the retrograde part of the plots denotes the abundance range of each element in $\omega$ Cen, as conservatively derived from the extreme values of the relatively small sample of stars analysed by Smith et al. (2000). The object symbols are used as described in Fig. 1. Two extreme objects are outside the $I_{z}$ range of this plot.

species. The exception is $\mathrm{Ca}$; however, we only have $\mathrm{Ca}$ values for three stars, from Adelman \& Philip (1996).

All stars in this group have orbits taking them to less than $1 \mathrm{kpc}$ of the Galactic centre - in some cases, to less than $300 \mathrm{pc}$. They are on chaotic orbits, which means that inclination and maximum distance from the plane changes with every revolution, and even small inaccuracies in the input values have a large effect on the shape (but not the overall size) of the orbit. Therefore the tangential velocities, $z_{\max }, n z e$, etc. do not really help in clarifying whether we are really dealing with a distinct group of stars; the eccentricity is over 0.9 for all stars, given their small perigalactic distances. Nonetheless, the evidence for this group is rather strong since we have these groupings in 7 of 8 element species, and especially in those where we have data for (nearly) all stars (Mg, Fe, Ti). In the near future we will be able to shed more light on the issue, when we have a consistent set of abundances for many elements.

A remarkable characteristic of the COG is that it goes so near the Galactic centre. Does this mean that it partook the initial collapse of the halo, as described in Eggen et al. (1962)? Does its small spread in chemical abundances indicate instead that it is a disrupted GC which was formerly on a radial orbit? Alternatively, this group could be the remains of yet another merging event, maybe one that took place in the relatively remote past (i.e., at least one revolution ago), and whose orbits have since decayed to the current cometary form. In the data of Peterson et al. (2001), who studied HBA stars in a window towards the bulge, we found a slight overdensity of stars with $[\mathrm{Fe} / \mathrm{H}] \sim-1.5$ (their values are only good to $0.5 \mathrm{dex}$ ) at a line of sight velocity of $100 \mathrm{~km} \mathrm{~s}^{-1}$ (Altmann 2002). In principle, 
these could be related to the stars discussed here; we would need the full kinematics (including accurate proper motions) to resolve this issue. This adds, however, to the evidence of inhomogeneities in the (inner) halo.

\section{Summary and outlook}

In this Letter, we have reported on the discovery of a group (the COG) of (local) HBA stars with similar (prograde) orbits and abundances, which may represent the remains of a protogalactic fragment. Their cometary orbits take the stars very close to the Galactic centre. The very small spread in abundances among the COG stars may alternatively support the possibility that they come from a disrupted GC. This group was discovered while we were testing a new method to search for debris of specific merger events associated with identified surviving remnants, such as $\omega$ Cen. The method uses orbital information and very detailed, multi-species abundance patterns to search for the chemical signatures of surviving merger remnants among (local) field stars having the appropriate kinematics. The rationale of the method is that a few key elements, such as $\mathrm{O}, \mathrm{Na}$, $\mathrm{Mg}, \mathrm{Al}, \mathrm{Cu}, \mathrm{Eu}$ and the s-process elements, may show unique signatures in the spotted surviving fragment (as has been seen in the case of $\omega$ Cen in particular), so that identifying field stars with similar abundances should represent the "smoking gun" indicating the presence of related tidal debris in the field.

More detailed abundances will both shed light into the case of a possible $\omega$ Cen remnant and further establish the reality and nature of the group dealt with in this Letter.

Acknowledgements. M.A. and M.Z. are supported by Fondap Center for Astrophysics 15010003, and M.C. by Proyecto FONDECYT Regular 1030954. We warmly thank M. Geffert and M. Odenkirchen for the kinematic software they readily supplied to us, and D. Dinescu, I. Ivans, B. J. Pritzl, and an anonymous referee for useful comments and discussions. With pleasure we made extensive use of the SIMBAD archive at CDS.

\section{References}

Adelman, S. J., \& Philip, A. G. D. 1996, MNRAS, 280, 285

Allen, C., \& Santillan, A. 1991, RMxA, 22, 255

Altmann, M. 2002, Ph.D. Thesis, Kinematics and Population Membership of BHB and EHB Stars, Sternwarte der Univ. Bonn Altmann, M., \& de Boer, K. S. 2000, A\&A, 353, 135

Altmann, M., Edelmann, H., \& de Boer, K. S. 2004, A\&A, 414, 181

Behr, B. B. 2003, ApJS, 149, 101

Bekki, K., \& Freeman, K. C. 2003, MNRAS, 346, L11

Dinescu, D. I. 2002, in Omega Centauri, A Unique Window into Astrophysics, ASP Conf. Ser., 265, 365

Eggen, O. J., Lynden-Bell, D., \& Sandage, A. R. 1962, ApJ, 136, 748 ESA. 1997, The Tycho and Hipparcos catalogue, SP-1200 (ESA)

Evans, D. S. 1967, in Determination of Radial Velocities and their Applications, IAU Symp., 30, 57

Ferraro, F. R., Sollima, A., Pancino, E., et al. 2004, ApJ, 603, L81

Gratton, R. G., Sneden, C., Carretta, E., \& Bragaglia, A. 2000, A\&A, 354, 169

Hilker, M., Kayser, A., Richtler, T., \& Willemsen, P. 2004, A\&A, 422, L9

Høg, E., Fabricius, C., Makarov, V. V., et al. 2000, A\&A, 355, L27

Ibata, R. A., Gilmore, G., \& Irwin, M. J. 1994, Nature, 370, 194

Kaempf, T. A., de Boer, K. S., \& Altmann, M. 2005, A\&A, 432, 879

Kinman, T., Castelli, F., Cacciari, C., et al. 2000, A\&A, 364, 102

Layden, A. C., \& Sarajedini, A. 2000, AJ, 119, 1760

Martin, N. F., Ibata, R. A., Bellazzini, M., et al. 2004, MNRAS, 348, 12

Mizutani, A., Chiba, M., \& Sakamoto, T. 2003, ApJ, 589, L89

Moehler, S. 2004, in The A-Star Puzzle, ed. J. Zverko, J. Ziznovsky, S. J. Adelman, \& W. W. Weiss (San Francisco: IAU), IAU Symp., 224,395

Norris, J., \& Bessel, M. S. 1975, ApJ, 201, L75

Norris, J. E., \& Da Costa, G. S. 1995, ApJ, 447, 680

Pancino, E., Pasquini, L., Hill, V., Ferraro, F. R., \& Bellazzini, M. 2002, ApJ, 568, L101

Peterson, R. C., Terndrup, D. M., Sadler, E. M., \& Walker, A. R. 2001, ApJ, 547, 240

Smith, V. V., Suntzeff, N. B., Cunha, K., et al. 2000, AJ, 119, 1239

Tsuchiya, T., Korchagin, V. I., \& Dinescu, D. I. 2004, MNRAS, 350, 1141 